\title{
Thermal Fluid Flow Transport Phenomenon in Unsteady Rotating Drum during Spin-Down Process
}

\author{
Shuichi Torii \\ Department of Mechanical Engineering and Materials Science, Kumamoto University, Kumamoto 860-8555, Japan \\ Email: torii@mech.kumamoto-u.ac.jp \\ Wen-Jei Yang \\ Mechanical Engineering and College of Engineering, The University of Michigan, Ann Arbor, MI 48109, USA \\ Email:wjyang@umich.edu
}

Received 10 March 2005

Numerical analysis is performed on unsteady-state thermal fluid flow transport phenomena in a rotating drum heated at constant wall temperature during a spin-down process. Emphasis is placed on the effect of aspect ratio of the drum on the velocity and temperature fields. The governing equations are discretized by means of a finite-difference technique and numerically solved to determine the distributions of velocity vector and fluid temperature in the drum. It is disclosed from the study that (i) the formation of Ekman boundary layers on both sidewalls of the drum results in a pair of recirculating flows, and (ii) an attenuation of the flow velocity is affected by the aspect ratio of a drum. Results may be used to develop a method to achieve uniform mixing in an enclosed vessel.

Keywords and phrases: spin-down, Ekman boundary layer, secondary flow, rotational effect, numerical analysis, rotating drum.

\section{INTRODUCTION}

Rotating cavities have been employed to model many practical rotating flows betweencorotating turbines or compressor discs (Owen [1]). The operationof rotating machinery experiences both the starting and stopping processes. In other words, such machinery is common to suddenly accelerate a rotating device from rest and to suddenly decelerate it from a steady rotation to rest.

Unsteady transport phenomena in hollow drum subjected to a sudden acceleration and deceleration were experimentally studied by means of laser doppler velocimetry (Kawashima and Yang [2]). The corresponding theoretical study was performed by Yang and Kawashima [3]. Later, they [4] observed flow pattern and temperature distribution in rotating drums subject to a sudden acceleration and inner surface heating, using the laser light-sheet method and ironconstantan thermocouples, respectively. In order to evaluate quantitatively the temperature distribution in the rotating drums, Kawashima and Yang [5] employed temperaturesensitive liquid crystals as a noninvasive method. A series of studies was focused on visualization of two-dimensional

This is an open access article distributed under the Creative Commons Attribution License, which permits unrestricted use, distribution, and reproduction in any medium, provided the original work is properly cited. thermal fields. To observe a three-dimensional hot plume growing inside a rotating system, Kawashima and Yang [5] employed the thermosensitive liquid crystal and thermocouple and investigated the formation and growth of thermally stratified layers. Ohue et al. [6] investigated energy dissipation and formation of the Ekman boundary layer in an unsteady flow during a spin-down process inside a rotating drum by means of both the tracer/light sheet and LDV methods. For further understanding of the mechanism for the thermal fluid-flow transport phenomenon, a numerical simulation is an effective alternate tool of investigation.

The present study deals with the unsteady thermal fluid flow transport phenomena in axially rotating drum under a spin-down process. Emphasis is placed on the effect of aspect ratio, $A$, on the velocity and temperature profiles. The governing differential equations for three-dimensional unsteady fluid flow are discretized by means of a finite-difference technique. Theoretical predictions, particularly the velocity fields, are compared with experimental results by Ohue et al. [6].

\section{GOVERNING EQUATIONS AND NUMERICAL METHOD}

Consider a forced flow through an axially rotating drum, which consists of cooled outer and heated inner cylinders. 


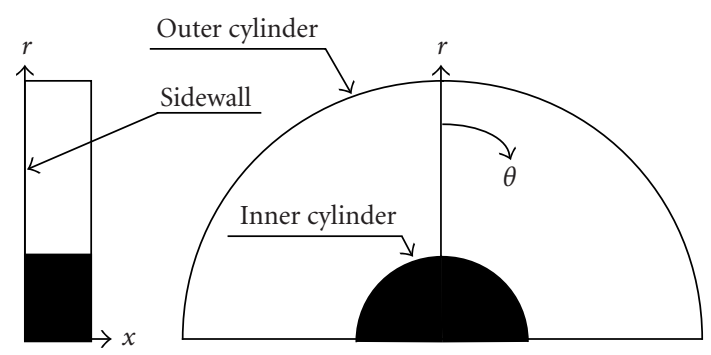

FIgURE 1: Schematic diagrams of axially rotating drum and their coordinates.

The physical configuration and the cylindrical coordinate system of the flow are shown in Figure 1. Nomenclature is summarized in Table 1. Here, the size of a drum is the same as Drum II employed by Ohue et al. [6], that is, it consists of an outer cylinderwith Dout $=240 \mathrm{~mm}$, an inner cylinder with Din $=80 \mathrm{~mm}$ and the width of $L=30 \mathrm{~mm}$. Thefollowing assumptions are imposed in the formulation of the problem based on the characteristics of the flow: it is an incompressible, laminar, unsteady flow with constant fluid properties. Then, the governing differential equations for mass, momentum and energy can be expressed as follows:

(i) continuity equation:

$$
\frac{\partial U_{i}}{\partial x_{i}}=0
$$

(ii) momentum equation:

$$
\frac{\partial U_{i}}{\partial t}+U_{j} \frac{\partial U_{i}}{\partial x_{j}}=-\frac{1}{\rho} \frac{\partial P}{\partial x_{i}}+\nu \frac{\partial}{\partial x_{j}}\left(\frac{\partial U_{i}}{\partial x_{j}}\right) ;
$$

(iii) energy equation:

$$
\frac{\partial T}{\partial t}+U_{i} \frac{\partial T}{\partial x_{i}}=a \frac{\partial}{\partial x_{i}}\left(\frac{\partial T}{\partial x_{i}}\right) .
$$

Here, the buoyancy force term is added in the momentum equation in the radial direction. An isothermal laminar flow in the absence of rotation is assumed as the inlet condition. Here, only one half of the $r-x$ cross section is calculated because of the symmetry of the fluid flow. The boundary conditions in the axially rotating drum are specified as follows:

\begin{tabular}{|c|c|}
\hline Symbol & Notation \\
\hline$A$ & Aspect ration, (Dout-Din)/2L \\
\hline$D_{\text {in }}$ & Diameter of inner cylinder in a drum, $\mathrm{mm}$ \\
\hline$D_{\text {out }}$ & Diameter of outer cylinder in a drum, $\mathrm{mm}$ \\
\hline$L$ & Drum width, mm \\
\hline$P$ & Time-averaged pressure, $\mathrm{Pa}$ \\
\hline $\operatorname{Pr}$ & Prandtl number \\
\hline$r$ & Radial coordinate, $\mathrm{m}$ \\
\hline$R$ & $\begin{array}{l}\text { Dimensionless radial distance, } \\
2 r /(\text { Dout-Din })\end{array}$ \\
\hline$T$ & time, $\mathrm{s}$ \\
\hline$U, V, W$ & $\begin{array}{l}\text { Time-averaged velocity components in axial, } \\
\text { radial, and tangential directions, } \\
\text { respectively, mm/s }\end{array}$ \\
\hline$U_{i}$ & $\begin{array}{l}\text { Velocity component in the } x_{i} \\
\text { Direction, } \mathrm{mm} / \mathrm{s}\end{array}$ \\
\hline$W_{\text {win }}$ & $\begin{array}{l}\text { Tangential velocity on inner wall, } D_{\text {in }} /(2 \omega) \text {, } \\
\mathrm{mm} / \mathrm{s}\end{array}$ \\
\hline$W_{\text {wout }}$ & $\begin{array}{l}\text { Tangential velocity on outer wall, } D_{\text {out }} /(2 \omega) \text {, } \\
\mathrm{mm} / \mathrm{s}\end{array}$ \\
\hline$x$ & Axial coordinate, $\mathrm{mm}$ \\
\hline$x_{i}$ & Coordinates, $\mathrm{mm}$ \\
\hline \multicolumn{2}{|c|}{ Greek letters } \\
\hline$v$ & Molecular viscosity, $\mathrm{mm}^{2} / \mathrm{s}$ \\
\hline$\rho$ & Density of fluid, Pasec \\
\hline$\omega$ & Angler velocity, $\mathrm{rad} / \mathrm{s}$ \\
\hline
\end{tabular}

$$
\begin{aligned}
& U=V=0, \quad W=W_{\text {win }}, \quad T=T w \quad \text { at inner cylinder wall, } \\
& U=V=0, \quad W=W_{\text {wout }}, \quad T=T c \quad \text { at outer cylinder wall, } \\
& U=V=0, \quad W=\frac{r}{\omega}, \quad \frac{\partial T}{\partial r}=0 \quad \text { at side wall }(x=0), \\
& \frac{\partial U}{\partial x}=0, \quad \frac{\partial V}{\partial x}=0, \\
& \frac{\partial W}{\partial x}=0, \quad \frac{\partial T}{\partial r}=0 \\
& \text { at center }\left(x=\frac{L}{2}\right) \text {. }
\end{aligned}
$$

TABle 1: Nomenclature.

To discretize the governing equations, the finite difference method is employed here. Note that this method is based on the conservative formulation, although the governing equations (1), (2), and (3) are written in nonconservative form. The system variables $P, U, V, W$, and $T$ are calculated with a staggered grid. Computation reveals only a slight difference when the grid system is properly changed from $120 \times 50 \times 30(r, \theta, x)$ to $240 \times 100 \times 60$, resulting in a gridindependent solution. Hence, a grid system of $120 \times 50 \times 30$ nodal points with uniformly distributed nodal points is employed here to also save computation time.

The numerical computation was performed on a personal computer, using water $(\operatorname{Pr}=7.1)$ as the working fluid filled in a drum. Numerical calculation was performed on the condition and drum size of experiments by Ohue et al. [6]. That is, the speed of rotation is 5 seconds per revolution.

Simulations with grids of various degrees of coarseness, as mentioned earlier, were conducted to determine the required resolution for grid-independent solutions. Throughout the numerical range considered here, the maximum error was estimated to be about $2 \%$ by comparing the solutions on regular and fine grids with twice the grid points. Although a few solutions were computed with half the time step to ensure consistency and time-step independence, there was no substantial discrepancy between two different time intervals. 


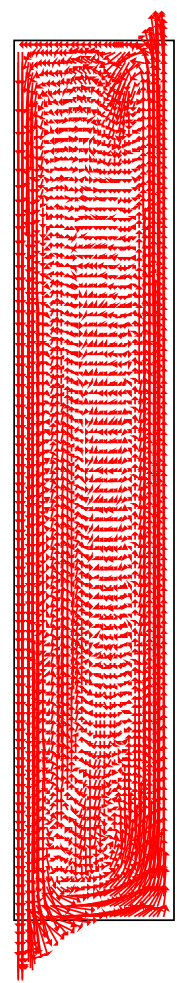

(a)

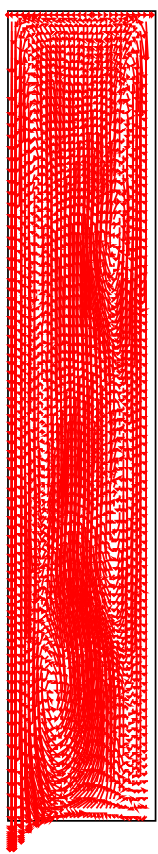

(b)

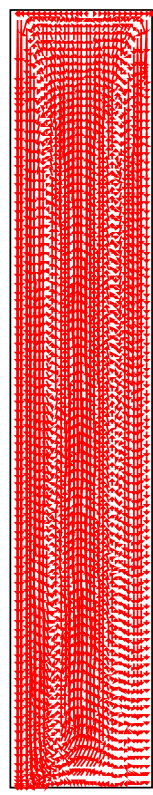

(c) (a)

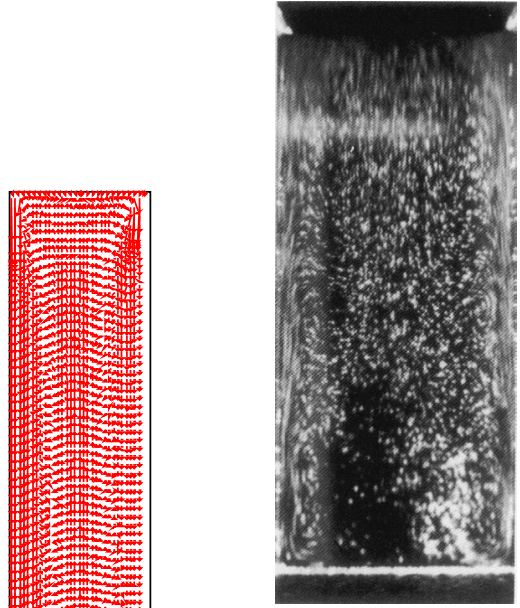

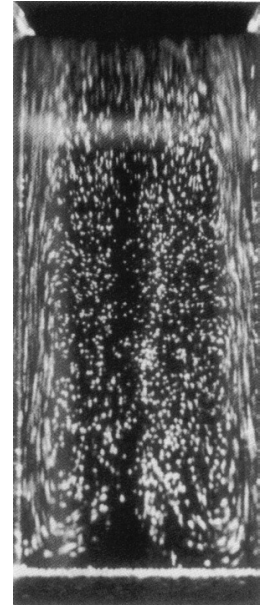

(b)

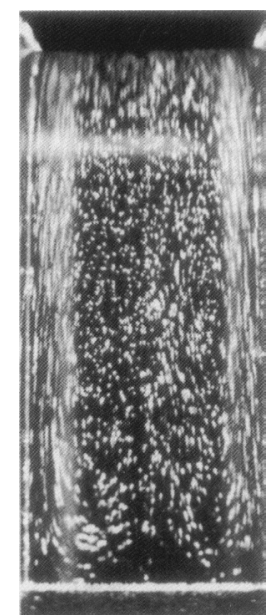

(c)

Figure 3: Photograph on meridional plane in drum under spindown process from $5 \mathrm{~s} / \mathrm{rev}$ to zero: (a) $t=6$ seconds, (b) $t=20$ seconds, and (c) $t=40$ seconds (Ohue et al. [6]).

flows in drum. It is found from Figures 2 and 3 that the radial fluid velocity in a drum during a spin-down process decreases with time with the formation of the Ekman boundary layer on the sidewall. This phenomenon becomes clearer in Figure 4 . Figure 4 depicts the radial velocity distribution at each radial location, in which experimental data of Ohue et al. [6] are superimposed for comparison. Figures $4 a, 4 b$, and $4 \mathrm{c}$ correspond to the experimental and numerical results at $t=6,20$, and 40 seconds, respectively. It is observed that the radially inward velocity near the sidewall is decreased toward the inner cylinder. A similar trend appears for different time of a spin-down process. As time progresses, the radial velocity at each radial location is diminished over an entire cross section of a drum. Figure 5 depicts the timewise variation of thermal field over the $r-x$ cross section in a drum with $A=2.7$ under a spin-down process from $5 \mathrm{~s} / \mathrm{rev}$ to zero. Figures $5 \mathrm{a}$ and $5 \mathrm{~b}$ correspond to numerical results for 6 and 40 seconds, respectively. Note that only one half of the $r-x$ cross section is depicted in the same form of Figure 2. Here, $\theta=1$ and 0 in Figure 5 correspond to the heated and cooled surfaces, respectively. In general,the shape of the isotherms resembles the flow structure. One observes that (i) the temperature gradient along the rising fluid flow increases in the upper region but decreases toward the bottom wall, and (ii) as time progresses, the thermal diffusion is suppressed. That is, the fluid temperature is diminished from the heated wall toward the cooled surface.

Next is to investigate the effect of aspect ratio, $A$, on the flow field in a drum. Figure 6 depicts the time history of the flow pattern over the $r-x$ cross-section in a drum with $A=1$ under a spin-down process from $5 \mathrm{~s} / \mathrm{rev}$ to zero. One observes that the radial velocity in the vicinity of the side wall is induced in the radial direction and the Ekman boundary layer appears as seen in Figure 6a. A largerecirculating flow results. As time progresses, the radial velocity diminishes faster near 


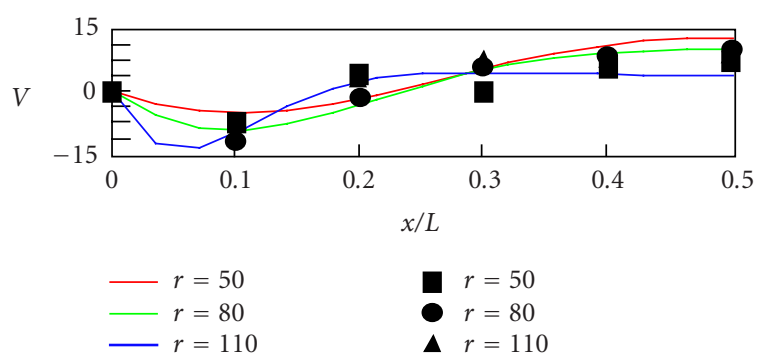

(a)
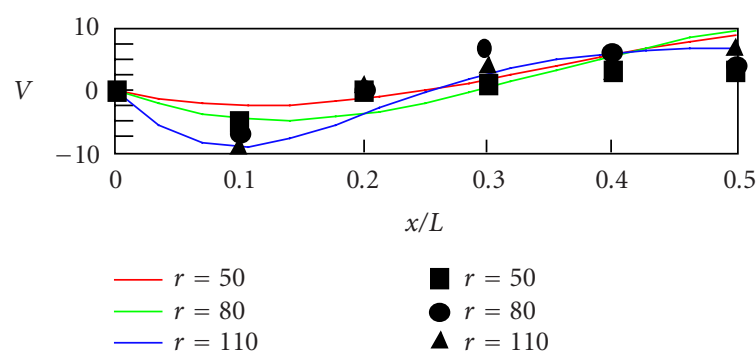

(b)

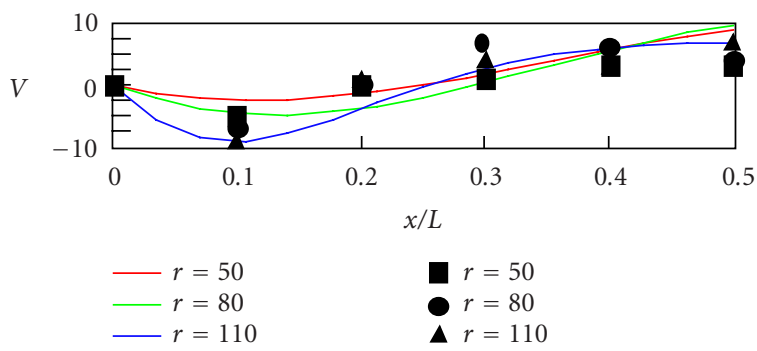

(c)

FIGURE 4: Velocity profiles on meridional plane in drum under spindown process from $5 \mathrm{~s} / \mathrm{rev}$ to zero: (a) $t=6$ seconds, (b) $t=20$ seconds, and (c) $t=40$ seconds (Ohue et al. [6]). Experimental data are presented by squares, circles, and triangles; prediction data by lines.

both the inner and outer cylinder walls than elsewhere due to sidewall effect, as seen in Figures $6 c$ and $6 d$. The attenuation of the velocity vector is somewhat lower than that in a rotating drum with $A=2.7$, as seen in Figure 2. This trend becomes clearer for velocity profiles. Figure 6 illustrates the velocitydistributions in the same form as Figure 4, in which the numerical results of Figure 4 are superimposed for comparison. Figures $7 \mathrm{a}, 7 \mathrm{~b}$, and $7 \mathrm{c}$ correspond to the radial velocity profile at a dimensionless radial location, that is, $R=0.5$ for $t=6,20$, and 40 seconds, respectively. It is found that at each time $t$, absolute value of the radial velocity is larger than that at $A=2.7$, so that the larger recirculating flow courses in the rotating drum with $A=1$. Since the radial velocity is suppressed due to sidewall effect, as mentioned previously, the side effect in the rotating drum with $A=1$ is lower than that at $A=2.7$. It is concluded from the study that, although the velocity distribution during a spin-down process of the

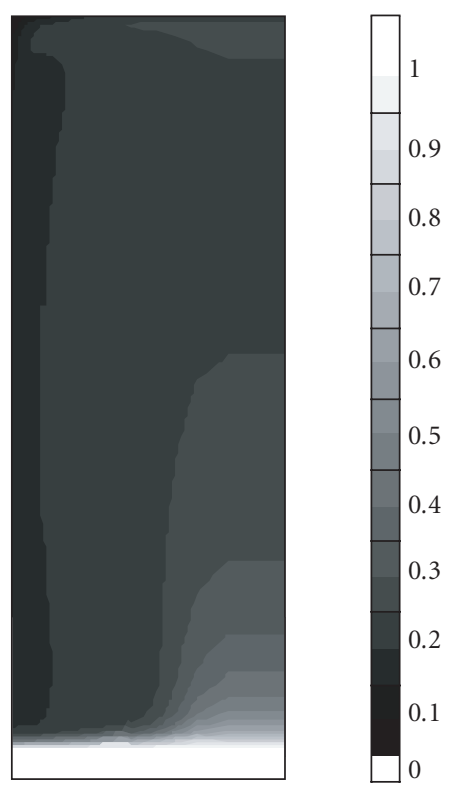

(a)

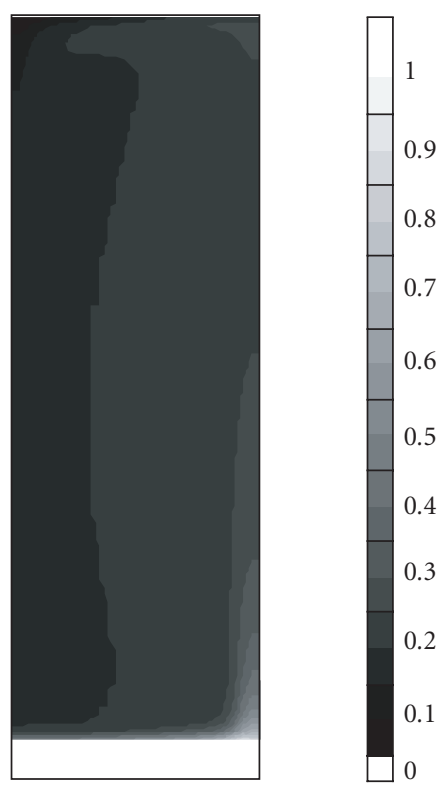

(b)

Figure 5: Thermal field on meridional plane in drum under spindown process from $5 \mathrm{~s} / \mathrm{rev}$ to zero: (a) $t=6$ seconds and (b) $t=40$ seconds.

rotating drum is affected by the aspect ratio, its effect is minor for the drum with a larger aspect ratio.

\section{SUMMARY}

Numerical simulation has been employed to investigate the fluid flow in an axially rotating drum under a spin-down process. The results are summarized as follows. The formation of 


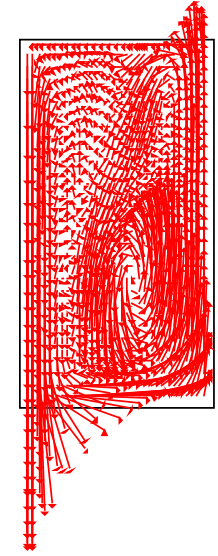

(a)

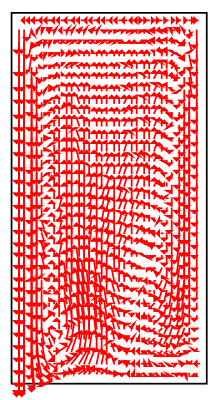

(b)

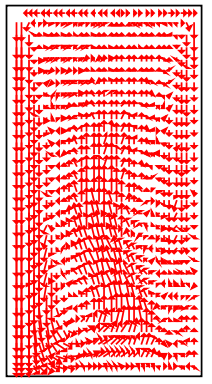

(c)

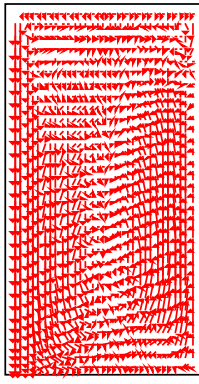

(d)

FIgURE 6: Velocity vector on meridional plane in drum with $A=1$ under spin-down process from $5 \mathrm{~s} / \mathrm{rev}$ to zero: (a) $t=0$ seconds, (b) $t=6$ seconds, (c) $t=20$ seconds, and (d) $t=40$ seconds.
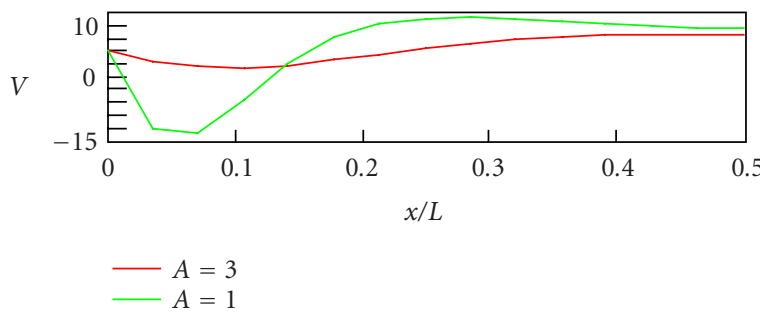

(a)

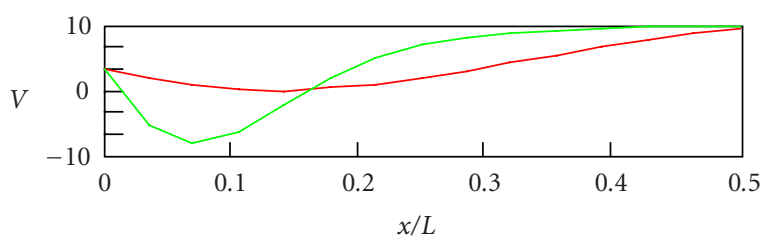

$A=3$

(b)

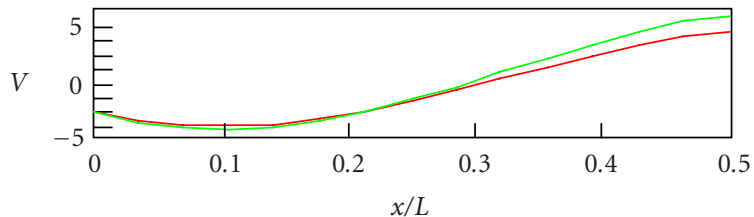

$-A=3$

(c)

Figure 7: Velocity profiles at dimensionless radial, $R=0.5$, in drums with two different aspect ratios under spin-down process from $5 \mathrm{~s} / \mathrm{rev}$ to zero: (a) $t=6$ seconds, (b) $t=20$ seconds, and (c) $t=40$ seconds (Ohue et al. [6]).
Ekman boundary layers on both sidewalls results in a recirculating flow, as seen inflow patterns and also evidenced in radial velocity distribution. This recirculating flow is somewhat larger for a drum with a relatively small aspect ratio. In other words, the effect of aspect ratio on the flow field is minor for a larger aspect ratio.

\section{REFERENCES}

[1] J. M. Owen, "Fluid flow and heat transfer in rotating disc systems," in Heat and Mass Transfer in Rotating Machinery, D. E. Metzger and N. H. Afgan, Eds., pp. 81-103, Hemisphere Publishing, Washington, DC, USA, 1984.

[2] G. Kawashima and W.-J. Yang, "Unsteady flow in rotating drums using laser doppler velocimetry," Experiments in Fluids, vol. 6, pp. 165-171, 1988.

[3] W.-J. Yang and G. Kawashima, "Hydrodynamics in unsteady rotating drums," in Transport Phenomena in Rotating Machinery, J. H. Kim and W.-J. Yang, Eds., pp. 51-61, Hemisphere Publishing, Washington, DC, USA, 1990.

[4] W.-J. Yang and G. Kawashima, "Experimental study on unsteady convection in rotating drums with inner surface heating," in Rotating Machinery - Transport Phenomena Machinery, J. H. Kim and W.-J. Yang, Eds., pp. 177-188, Hemisphere Publishing, Washington, DC, USA, 1992.

[5] G. Kawashima and W.-J. Yang, "Natural convection in rotating drums with inner surface heating," Experimental Heat Transfer, vol. 6, no. 3, pp. 287-297, 1993.

[6] H. Ohue, G. Kawashima, and W.-J. Yang, "Ekman boundary layers and energy dissipation in rotating drums during spindown process," International Journal of Rotating Machinery, vol. 2, no. 2, pp. 113-121, 1995. 

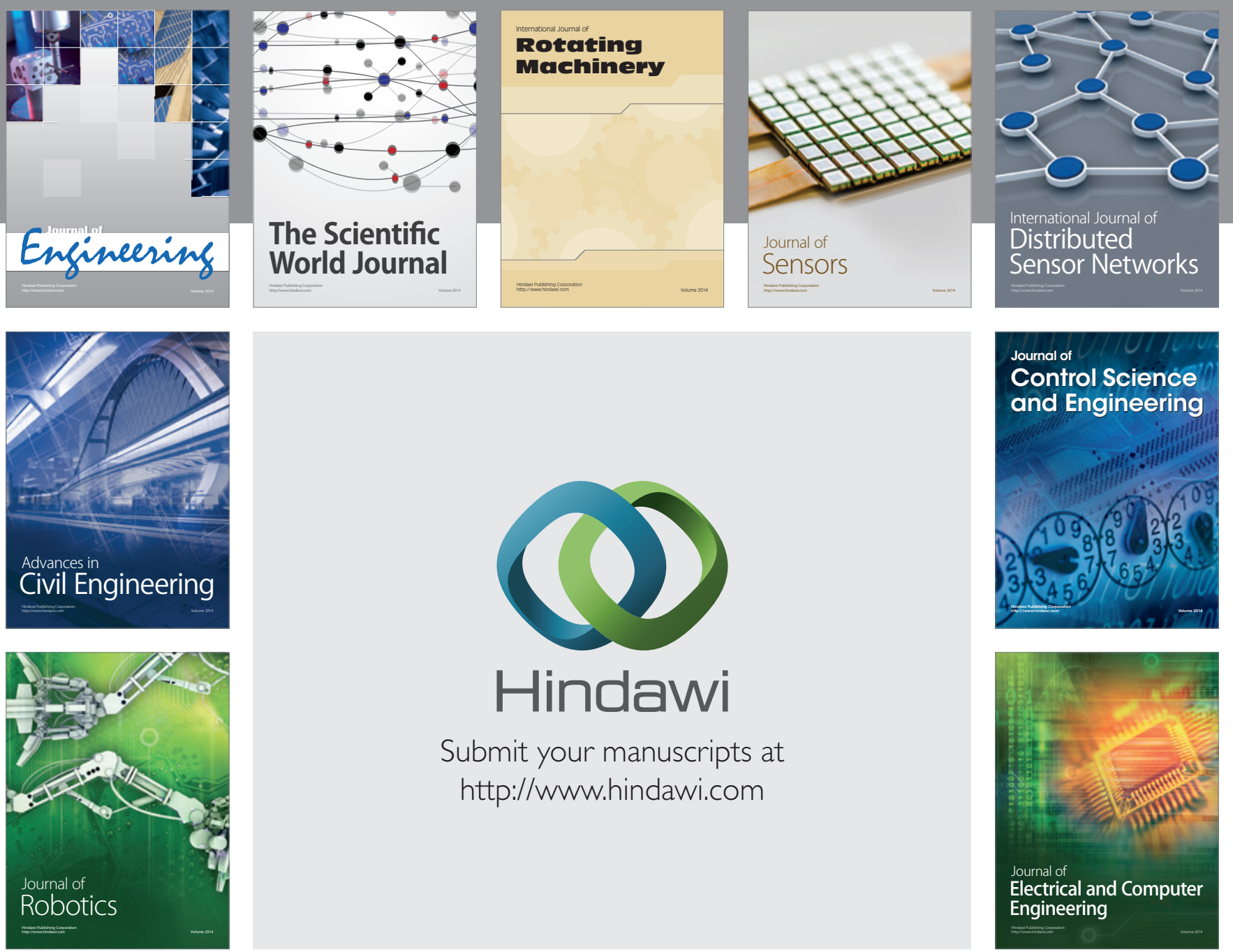

Submit your manuscripts at

http://www.hindawi.com
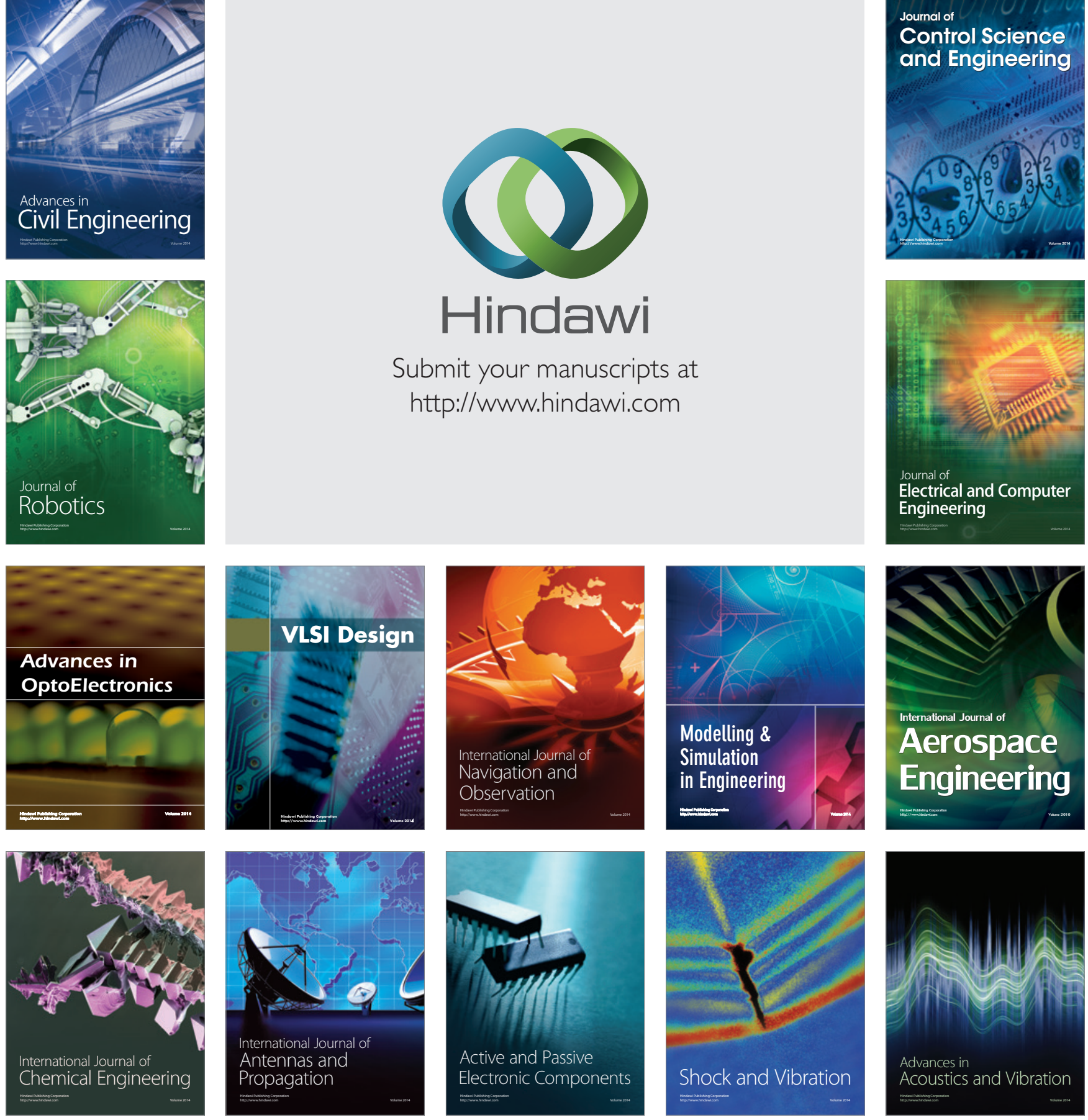